\title{
A Service-Oriented Architecture based Global Positioning System
}

\author{
Mohammed Jaleeluddin ${ }^{1}$, Mahe Jabeen ${ }^{2}$, Dr.D. Vijayalakshmi ${ }^{3}$ \\ ${ }^{1}$ Research Scholar, Jawaharlal Nehru Technological University Hyderabad, INDIA \\ ${ }^{2}$ Shadan Women's College of Engineering and Technology, JNTU Hyderabad, INDIA \\ ${ }^{3}$ Professor and H.O.D, Dept. Of Information Technology, MGIT, JNTU Hyderabad, INDIA
}

\begin{abstract}
The Global Positioning System (GPS) is a space-based satellite navigation system that provides location and time information, anywhere on or near the Earth. The GPS provides critical capabilities to military, civil and commercial users around the world. This paper presents a Service oriented architecture (SOA) based Global Positioning System (GPS). In case of military and civilian applications of GPS, exchange of information is not that easy as it becomes perilous. There should be a qualified and well standard approach that can be used for the secure communication. A detailed architecture has been used to justify the essential need for the proposed architecture. Service oriented architecture provides communication with flexible, secure and independent platforms and standards making it a strongly viable option for secure communication when needed.
\end{abstract}

Keywords—Service Oriented Architecture, Global Positioning System, Enterprise Service Bus

\section{INTRODUCTION}

A service-oriented architecture (SOA) is a set of principles and methodologies for designing and developing software in the form of interoperable services. These services are well-defined business functionalities that are built as software that can be reused for different purposes. SOA generally provides a way for consumers of services, such as web-based applications (through mobile or tablet or PC), to be aware of available SOA-based services. For example, several users may develop and deploy SOA services in different implementation languages; their respective clients will benefit from a well-defined interface to access them. $\mathrm{XML}$ is often used for interfacing with SOA services, though this is not mandatory. Enterprise Service Bus is used for the transformation of messages, message enrichment, protocol mediation and routing to all existing services involved in the information exchange.

The Global Positioning System (GPS) is a space-based satellite navigation system that provides location and time information in all weather, anywhere on Earth, where there is an unobstructed line of sight to four or more GPS satellites. It is maintained by the United States government and is freely accessible to anyone with a GPS receiver. This paper presents a proposed service oriented architecture which uses Enterprise Service Bus (ESB) to respond to the requests made by the users which involves exchange of information such as location and time.

\section{WHY SOA?}

This concept is based on an architectural style that defines an interaction model between three primary parties: the service provider, who provides the implementation for the service, a service consumer, who invokes the service. The service broker provides and maintains the service registry, although registries are not mandatory.

Rather than defining an API, SOA defines the interface in terms of protocols and functionality. These services and their corresponding consumers communicate with each other by passing data in a well-defined, shared format, or by coordinating an activity between two or more services. SOA separates functions into distinct units, or services, which developers make accessible over a network in order to allow users to combine and reuse them among various applications. An Enterprise Service Bus (ESB) is an architectural construct and middleware infrastructure component that supports Service-Oriented Architectures (SOA). Enterprise Service Bus is used for the transformation of messages, message enrichment, protocol mediation and routing to all existing services involved in the information exchange. Business Process Execution Language (BPEL) provides a language for the specification of Executable and Abstract business processes. By doing so, it extends the Web Services interaction model and enables it to support business transactions. It defines an interoperable integration model that should facilitate the expansion of automated process integration both within and between businesses. 
The first published research of service orientation from an industry perspective was provided by Thomas Erl of SOA Systems who defined eight specific service-orientation principles common to all primary SOA platforms.

- Standardized service contract: Services adhere to a communications agreement, as defined collectively by one or more service-description documents.

- Service loose coupling: Services maintain a relationship that minimizes dependencies and only requires that they maintain an awareness of each other.

- Service abstraction: Beyond descriptions in the service contract, services hide logic from the outside world.

- Service reusability: Logic is divided into services with the intention of promoting reuse.

- Service autonomy: Services have control over the logic they encapsulate.

- Service statelessness: Services minimize resource consumption by deferring the management of state information when necessary

- Service discoverability: Services are supplemented with communicative meta data by which they can be effectively discovered and interpreted.

- Service composability: Services are effective composition participants, regardless of the size and complexity of the composition. [3]

It is because of the above principles that adopting SOA for GPS will prove to be the best possible and optimal solution.

\section{GLOBAL POSITIONING SYSTEM(GPS)}

\subsection{Basic Concept of GPS}

A GPS receiver calculates its position by precisely timing the signals sent by GPS satellites high above the Earth. Each satellite continually transmits messages and the receiver uses the messages it receives to determine the transit time of each message and computes the distance to each satellite using the speed of light. These distances along with satellites' locations define several spheres. The receiver is on the surface of each of these spheres when the distances and the satellites' locations are correct. These locations are used to compute the location of the receiver. This location is then displayed, perhaps with a moving map display or latitude and longitude; elevation information may be included. Many GPS units show derived information such as direction and speed, calculated from position changes. [2]

\section{The main characteristics of GPS are:}

GPS only listens to signals from satellites - it does not transmit any signals itself. This is very important to the military, since GPS can then be used without giving away the observers location.

GPS needs to receive signals from at least four different satellites to get a precise fix. This is why GPS often works poorly when surrounded by tall obstructions, and cannot work at all in tunnels or other locations where the satellites are not visible.GPS calculates a (very accurate) time as well as a location. This time is unused in many GPS applications, but important to a few, including time transfer, traffic signal timing, and synchronization of cell phone base stations.

The current GPS consists of three major segments. These are the space segment (SS), a control segment (CS), and a user segment (US).The U.S. Air Force develops, maintains, and operates the space and control segments. GPS satellites broadcast signals from space, and each GPS receiver uses these signals to calculate its three-dimensional location (latitude, longitude, and altitude) and the current time [2].

\section{SCOPE OF GPS}

GPS is an essential element of the global information infrastructure. The free, open, and dependable nature of GPS has led to the development of hundreds of applications affecting every aspect of modern life. GPS technology is now in everything to every field. GPS boosts productivity across a wide range of the economy, including farming, construction, mining, surveying and logistical supply chain management. Major communications networks, banking systems, financial markets, and power grids depend heavily on GPS for precise time synchronization. GPS saves lives by preventing transportation accidents, helping search and rescue efforts, and speeding the delivery of emergency services and disaster relief. GPS is vital to the Air Transportation System that will enhance flight safety while increasing airspace capacity. GPS also advances scientific events such as weather forecasting, earthquake monitoring, and environmental protection.

\section{Aviation}

Aviators throughout the world use the Global Positioning System (GPS) to increase the safety and efficiency of flight. GPS offers seamless satellite navigation services that enable 3D position determination for 
all phases of flight from departure, en route, and arrival, to airport surface navigation. GPS helps in air traffic management system and aircraft tracking system

\section{Environment}

In order to maintain the Earth's environment while balancing human needs requires better decision making with up-to-date information. Gathering accurate and timely information has been one of the greatest challenges facing both government and private organizations that must make these decisions. The modernization of GPS will further enhance the support of GPS technology to the study and management of the world's environment.

\section{Agriculture}

The development and implementation of precision agriculture or site-specific farming has been made possible by combining the Global Positioning System (GPS) and geographic information systems (GIS). These technologies enable the coupling of real-time data collection with accurate position information, leading to the efficient manipulation and analysis of large amounts of data.

\section{Marine}

GPS is very helpful for marine operations like search and rescue. GPS provides the fastest and most accurate method for mariners to navigate, measure speed, and determine location of the ships. This enables increased levels of safety and efficiency for mariners worldwide.

\section{Emergency \& Disaster Relief}

Time is a very critical component of any successful rescue operation .Knowing the precise location of landmarks, streets, buildings, emergency service resources, and disaster relief sites reduces that time and saves lives. This information is critical to disaster relief teams and public safety personnel in order to protect life and reduce property loss. Thus GPS serves as a facilitating technology in addressing these needs.

\section{Railways}

Rail systems throughout the world use GPS to track the movement of locomotives, rail cars and maintenance vehicles in real time. When combined with other sensors, computers, and communications systems, GPS improves rail safety, security, and operational effectiveness. The technology helps to reduce accidents, delays as well as operating costs and thus increases customer satisfaction and cost effectiveness.

\section{Recreation}

GPS has eliminated many of the hazards associated with common recreational activities by providing a capability to determine a precise location. GPS receivers have also broadened the scope and enjoyment of outdoor activities by simplifying many of the traditional problems, such as staying on the right path. GPS tours can either be GPS guided or self-directed tours that provide visitors with relevant content about location of interest along a route.

\section{Roads \& Highways}

The estimate shows that the delays due to congestion on highways, streets, and transit systems throughout the world result in productivity losses in the crores of money annually. Other negative effects of congestion include property damage, personal injuries, increased air pollution, and inefficient fuel consumption. The availability and accuracy offers increased efficiency and safety for vehicles using highways and streets. Many of the problems associated with the routing and dispatch of commercial vehicles is significantly reduced with the help of GPS.

\section{Space}

GPS is revolutionizing and the way nations operate in space, from guidance systems for crewed vehicles to the management, tracking, and control of communication satellite constellations, to monitoring the Earth from space. Benefits of using GPS include Navigation solutions, Attitude solutions, Timing solutions, Constellation control, Formation flying, Virtual platforms and Launch vehicle tracking. [3]

\section{Mapping and Surveying}

Today, GPS is a vital part of surveying and mapping activities around the world .GPS-based data collection is much faster than conventional surveying and mapping techniques, reducing the amount of equipment and labor required. 


\section{SOA BASED GLOBAL POSITIONING SYSTEM}

The SOA Based Global Positioning System is shown in fig 1.A global positioning system based on SOA contains n number of service consumers, an Enterprise Service Bus and ' $n$ ' Service Providers. A Service Consumer in this architecture can be any application requesting data through the GPS. Service consumers can be Web browsers on Internet, mobile phones or any other GPS portals. .Service Providers includes the GPS receivers. GPS receivers come in a variety of formats, from devices integrated into cars, phones, and watches, to dedicated GPS devices.

Service consumers can invoke different services which are exposed by Enterprise service Bus through web services. These Services will be classified as military Services and Civilian Services. Military Services includes navigation service, Target tracing system service, Missile \& Project guidance service, Search \& rescue Service, Reconnaissance service and nuclear detonation and detection service. Civilian Services includes Clock Synchronization Service, Cellular Telephony Service, Disaster relief and Emergency Service, Aircraft Tracking System Service, Geofencing Service, Railways Service, Roadways Service, Marine Service, Surveying and Mapping Service, Techtonics Service and Telematics Service.

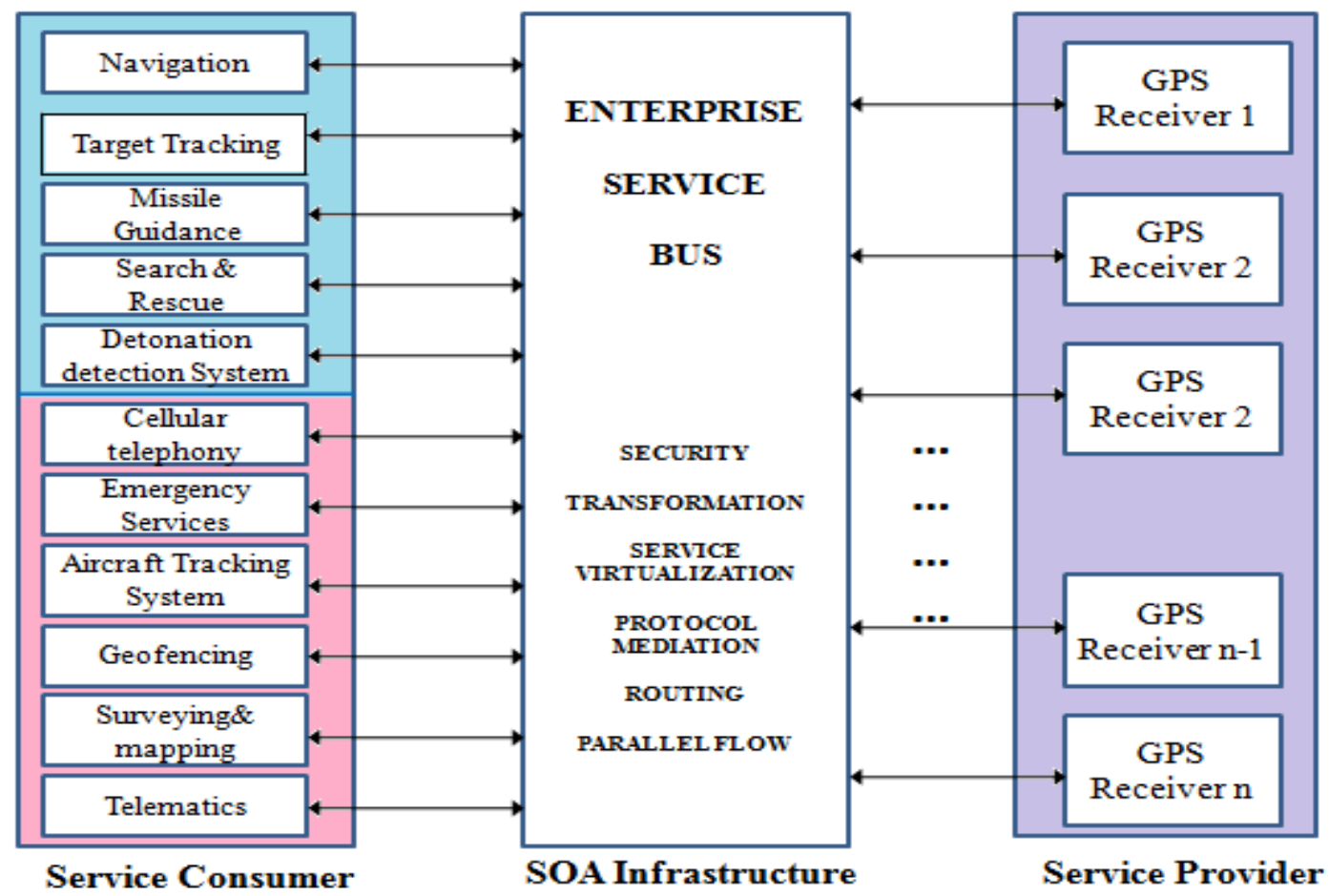

Figure 1 SOA Based Global Positioning System

Enterprise Service Bus (ESB) is a mediator between service consumers and service providers. ESB receives the request from service consumers, validates the request, transforms the request, and routes the request to the designated service provider or GPS receiver. GPS Receiver sends the results back to ESB. ESB transforms, enriches the response and sends back to service consumers in the same transaction. ESB encapsulates and abstracts the service provider and service implementation details to Service consumers. Service Consumers are unaware of the location and other details of the service providers thus minimizing the dependency between service consumers and service providers. Service providers can be added or removed dynamically without the knowledge of service consumers. Service consumers can be added or removed without the knowledge of service providers.

Enterprise Service Bus provides reliable, scalable and easy to manage clustering and load balancing to ensure high availability. Enterprise Service Bus provides security to protect the services from unauthorized use.

\section{Layered Architecture for SOA-based GPS System:}

The layered architecture of SOA-based GPS System consists of four different layers. This is shown in the Fig 2.As shown in the figure; the first layer is the interface layer. Interface layer provides interface for users to interact with the system. Since SOA is applied, the interface depends on different clients called by ESB services. As a result, this layer may include mobiles, PC's, laptop's, web browsers, GPS Tracking Systems and Navigation devices. 


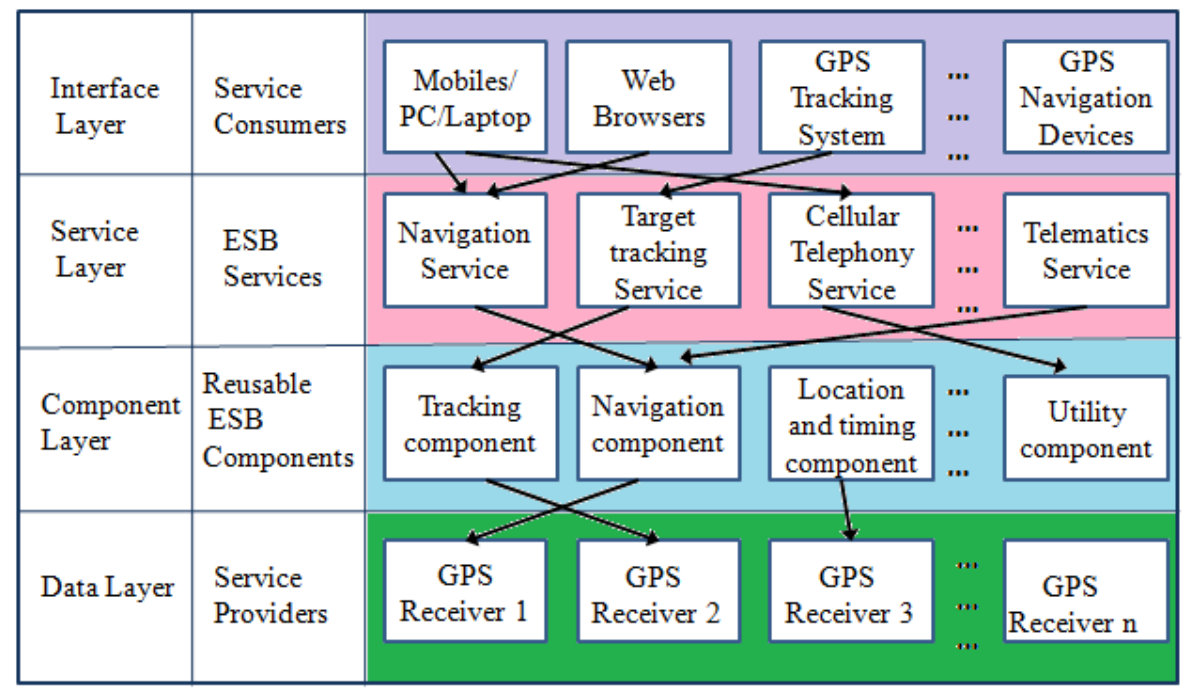

Figure2 Layered Architecture

The next layer is the service layer. It consists of different services provided by the ESB to the service consumers. It may include services for both military and civilian applications. These may be navigation services, target tracking and other services for military applications. In case of civilian applications some of the services are Cellular Telephony Services, Telematics services and other tracking services. The component layer consists of reusable ESB components such as components for tracking system, navigation systems, location and timing or any utility component. The bottom layer is the data layer which consists of service providers which can be any GPS receiver.

\section{CONCLUSION \& FUTURE SCOPE}

Combining SOA in a GPS system, this paper explores the advantages of SOA-based GPS System and layered architecture which focuses on how this works. Employing this proposed architecture for GPS System gives the advantage of service loose coupling, service reusability and service composability making it a feasible option. In future, more components can be reused, a complex business process layer can be used with the help of WS-BPEL specifications and RESTful services can be employed in SOA to achieve scalability and independency of the components. A model for Unified SOA Governance system for SOA-based GPS system needs to be proposed and implemented to get maximum benefits of Service Oriented Architecture.

\section{REFERENCES}

[1] Service -Oriented Architecture, Concepts, Technology and Design" by Thomas Erl

[2] http://en.wikipedia.org/wiki/Global_Positioning_System

[3] http://www.gps.gov/

[4] http://innovation.in.csc.com/contest/uploads/mjaleeluddin@csc.com_Jaleeluddin_SOADDM.docx

\section{AUTHORS PROFILE}

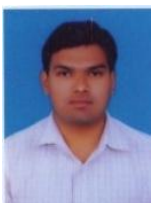

Mohammed Jaleeluddin Received B.Tech in Computer Science and Engineering from MGIT, JNTU Hyderabad and M.Tech in Computer Science from JNTU College of Engineering, Hyderabad, JNTUH. He is presently pursuing Ph.D in Computer Science from JNTU Hyderabad. He is working as a Senior Consultant in Computer Sciences Corporation (CSC) India. His research interest includes Service Oriented Architecture (SOA), GPS, Cloud Computing, Data Mining, EAI, BPEL, BPM and ESB.

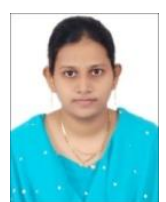

Mahe Jabeen did her B.Tech in Electronics and Communication Engineering and M.Tech in VLSI Systems Design from Shadan Women's College of Engineering and Technology, JNTU Hyderabad. She has 3 years of industrial experience in IBM India Private Limited. Her research interest includes Satellite Communications, VLSI Design, Global Positioning System, GPRS and Digital communication systems.

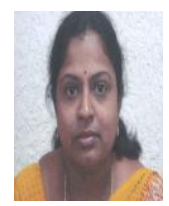

Dr. D. Vijayalakshmi holds Ph.D in Computer Science and Engineering from JNTU Hyderabad. Currently, she is working as Professor and Head of Department of Information Technology, MGIT, Hyderabad. She has 18 years of professional teaching experience. Her research interest includes GPS, GPRS, GIS, GSM, SOA, Data Mining, Cloud Computing and satellite communications. 